\title{
Mechanisms of Action of Proteins, Peptides and Biomarkers in Single Cell Analysis
}

John WS Ho*

Associate Professor, School of Life Sciences, Chinese University of Hong Kong, Hong Kong

Cell biology is an important area of research. With the advent of bio-chemical and molecular methods, technologies and protocols for cell biology research, important cloning reactions, signaling processes, cellular activities and mechanism of actions of extrinsic and intrinsic molecules can be monitored and measured. Different technologies provide tools to help researchers build a foundation of platforms to conduct research in different perspectives and to better understand cell biology in particular cancer biology ranging from gene expression to function. These findings can be used to drug development for cancer therapy with a molecular target and consequently, it would improve treatments for cancer with new drugs. For a single cell, high-content imaging systems for the study target cells and their components are proven tools. In addition, improved cell technologies offer innovative solutions for production of different cell lines and the measurement of gene products. If potential target proteins for inhibitors and/or activators are to be determined, an overexpression and purification of these proteins is the next logical step. The findings enable researchers to identify differentially expressed proteins as a result of cellular response to drug treatment. In addition to the local changes in target tissues or an inflammatory area, there are often systemic manifestations of inflammatory disease, for example an increase in leucocytes or neutrophils and the release of associated proteins from the liver indicate cellular responses to external stimuli. For example, the level of C-reactive protein, fibrinogen and $\alpha_{1}$-antitrypsin would change. While function of many of these cellular components remains sketchy, they all seem to have defense actions. Some of these proteins scavenge iron or block proteases to protect the host against the external stimuli including oxidative stress.

The recent discovery in which single human cells can be derived from somatic cells through the expression of exogenous genes has produced great excitement and expectation in the fields of reproductive biology and genetic engineering. These induced cells including stem cells have been used to further our understanding of basic cellular responses and the sequential activity in an organism. As a result, new therapeutic drugs and treatment of illness through cell therapy can be developed. Stem cell treatment is an intervention strategy that enables stem cells to get in the damaged tissue as a new approach for treatment of disease or injury. It is believed that the strategy could change the convention therapy and alleviate suffering. The understanding of stem cell biology allows subsequent generations with variable degrees of cell differentiation capacities. It offers tremendous potential for tissue generation in the way the new tissue can replace diseased and the damaged organelles or organs in the body. The discovery offers significant potential treatment with less risk of rejection and undesirable effects. Although a number of stem cell therapies exist, they are at experimental stages and indeed the technology needs to be improved. It is expected that embryonic stem cells can be used to treat cancer and Type 1 diabetes mellitus, cardiac failure, Huntington's disease and neurological disorders including Parkinson's disease and many other diseases such as spinal cord injury, heart damage, baldness, blindness and vision impairment, birth defects and infertility. However, before cell therapies can be used in clinical trial, more research is necessary to understand stem cell biology and their activity in transplantation. More importantly, the mechanisms of stem cell interaction with diseased or damaged tissues need to be unfolded. The study of single cell biology offers significant advantages in understanding the interrelated signaling process in an organism. Monitoring of the activity of single cells does not present any ethical barriers for use in research and cell therapy. A broad technology platform provides sensitive tools from isolation and characterization of different cell types to the validation of cellular mechanisms of differentiation. The technology platform including signal amplification technology allows researchers to experiment on all aspects of single cell research. The measurement of changes in mitochondrial DNA is important for comparing biological processes in different cell types. The results are implicated in drug toxicity, aging and various diseases.

Cellular heterogeneity is attributed to the expression of genes, proteins and metabolites. It forms the fundamental principle of cell biology, but single cell analysis has been beyond the capability of convention technology. To cope with the advances and the rapidly changing science with the recent examples of single cell genomics, transcriptomics, proteomics and metabolomics, more new platforms and tools for single cell research would be helpful. Molecular technology interfaces for mass spectrometry and automated DNA sequencers enable researchers to fathom out the mystery of the cell. Single cell analysis is the new frontier in "OMICS"-era, which has the potential to transform cell biology through new discoveries derived from cellular heterogeneity.
${ }^{*}$ Corresponding author: John WS Ho, Associate Professor, School of Life Sciences, Chinese University of Hong Kong, Hong Kong, E-mail: ws203ho@cuhk.edu.hk

Received August 24, 2012; Accepted August 27, 2012; Published August 30, 2012

Citation: Ho JWS (2012) Mechanisms of Action of Proteins, Peptides and Biomarkers in Single Cell Analysis. Single Cell Biol 1:e115. doi:10.4172/21689431.1000e115

Copyright: (c) 2012 Ho JWS. This is an open-access article distributed under the terms of the Creative Commons Attribution License, which permits unrestricted use, distribution, and reproduction in any medium, provided the original author and source are credited. 